\title{
A Prospective Randomised Comparative Study between Clip Less Versus Conventional Laparoscopic Cholecystectomy
}

\author{
Dr. Pallem Praveen ${ }^{\#}$ and Dr. Ajmeera Ranga^ ${ }^{*}$ \\ \#MS General Surgery, Associate Professor, ^Assistant Professor of Surgery, Gandhi Hospital, Musheerabad, Secunderabad, Telangana, India
}

Received 01 Dec 2017, Accepted 02 Feb 2018, Available online 07 Feb 2018, Vol.6 (Jan/Feb 2018 issue)

\begin{abstract}
Introduction: For the surgical treatment of gall bladder diseases, laparoscopic Chole cystectomy has been accepted as the gold standard. The minimally invasive procedure is undeniably superior in various respects when compared with open surgery and this is also true on the aesthetic criteria when the conventional laparoscopic cholecystectomy (CLC) is compared with the mini-laparoscopic cholecystectomy (MLC).

Objective: Evaluate the hospital charges associated with these procedures and specify the differences concerning these techniques.

Method: Comparative and retrospective study of hospital charges, with 40 consecutive patients, who underwent laparoscopic cholecystectomy.

There were two groups with 20 patients each. One group underwent conventional laparoscopic cholecystectomy and in the other the minimally invasive approach was performed. The arithmetic mean was used to compare the total charges for the entire procedures.

Results: The MLC procedures showed no significant difference in total hospital charges compared to the CLC approach.

Conclusion: The equivalence of hospital charges for the two procedures suggests that the mini-laparoscopic cholecystectomy (MLC) should be widely recognized among surgeons as offering better aesthetic results the conventional laparoscopic procedure. Studies comparing patient satisfaction with the surgical result, difference in postoperative morbidity, pain, and recuperation for the two procedures are needed.
\end{abstract}

Keywords: charges, surgery, laparoscopy, needlescopic instruments

\section{Introduction}

Ever since the first laparoscopic procedure, the advantages and indications for this technique have increased systematically [1]. For the surgical treatment of diseases of the gallbladder, laparoscopic cholecystectomy has become the gold standard around the world. Now, mini-laparoscopic cholecystectomy - which is quite effective for removing the gall bladder - is growing rapidly in popularity among surgeons. Because it provides aesthetic results similar to those with NOTES (natural orifice transluminal endoscopic surgery), it is being hailed as a new phase in videosurgery $[1,3]$.

The superiority of minimally invasive procedures when compared with open surgery in various aspects is undeniable, and this is also true between conventional laparoscopic cholecystectomy and mini laparoscopic cholecystectomy when you refer to aesthetic aspects [2]. Incision diameters that are significantly reduced, resulting in imperceptible scars would be reason enough to justify

*Corresponding author's ORCID ID: 0000-0000-0000-0000 DOI: https://doi.org/10.14741/ijmcr.v6i01.10915 the mini-laparoscopic procedure, but in addition to this, there appears to be less postoperative pain, resulting in greater patient satisfaction. These facts support the need for greater dissemination and the indication of the minilaparoscopic cholecystectomy for more patients [4, 5].

Table 1 Historical lamdmarks in development of laproscopic adrenalectomy

\begin{tabular}{|c|c|c|}
\hline \multirow{2}{*}{$\begin{array}{c}\text { Laproscopic } \\
\text { adrenalectomy }\end{array}$} & Authors & Year \\
\hline $\begin{array}{c}\text { LA fo } \\
\text { phaeochromcytoma }\end{array}$ & Ganger et al et al & 1992 \\
\hline \multirow{2}{*}{ Bilateral LA } & Ganger et al & 1992 \\
\cline { 2 - 2 } & Ferandez & \multirow{2}{*}{1993} \\
\cline { 2 - 2 } & Criz el al & 1997 \\
\hline LA formalignacy & Ushiyama et al & 1998 \\
\hline $\begin{array}{c}\text { Needlescopic LA using } \\
2 \text { mm instruments }\end{array}$ & Ganger et al & \multirow{2}{*}{1998} \\
\hline Day care LA & Edwin et al & 2001 \\
\hline
\end{tabular}

Still, changing paradigms or surgical techniques, involve overcoming historically enormous barriers and taboos; 104 | Int. J. of Multidisciplinary and Current research, Vol.6 (Jan/Feb 2018) 
such changes are part of the evolution of surgical technique, of the innovation of procedures, and the technological advances in health. Moreover, the change in surgical technique proposed here, involves not only the greater dexterity on the part of the surgeon in handling the delicate equipment, but also the purchase of this expensive equipment, and time-consuming training.

Unequivocally, underwriting the costs of this new technique, by either the patient or the hospital, is mandatory for the success and diffusion of the procedure $[6,7]$. Given the dearth of studies comparing the costs of conventional laparoscopic cholecystectomy (CLC) and the mini-laparoscopic cholecystectomy (MLC), this study sought to evaluate the hospital charges associated with these procedures, and also specifies the difference in hospital charges of the surgical techniques, and the implications for the total cost of the procedure.

\section{Patients and methods}

This is a retrospective comparative study, of 40 consecutive patients, who underwent laparoscopic cholecystectomy. Twenty patients were operated by a single surgeon using the conventional laparoscopic cholecystectomy $(\mathrm{CLC})$ technique, and 20 patients were operated by another surgeon using the mini-laparoscopic cholecystectomy (MLC) technique.

All the patients were considered to have been hospitalized on a nursing ward, with use of the anesthesia recovery room for up to six hours and having utilized capnography, infusion pumps, and oxygen during the hospitalization. In addition, because the cases were accumulated over a period of 18 months, all charges were adjusted so that there were no price increases over time for the items charged.

Only hospital charges were considered, covering the period of the hospitalization, and were obtained from the hospital bill for each surgery. After all the bills were evaluated, adjustments were made to the charges in order to standardize them as described above, and a spreadsheet was developed in order to compare the charges of each step of procedures. In this way it was possible to arrive at an average total charge for the procedures in the two groups studied.

\section{Operative techniques}

\section{Conventional Laparoscopic Cholecystectomy}

After standard positioning of the surgical team (Figure 1), the pneumoperitoneum was established by the closed technique with a Veres needle, using an umbilical incision, through which a $10 \mathrm{~mm}$ trocar was inserted, attaining an intra-abdominal pressure of 10 to $14 \mathrm{mmHg}$. After the pneumoperitoneum was established a $30 \% / 10 \mathrm{~mm}$ optic was introduced through the umbilical trocar.

Three more trocars were then inserted (Figure 2A): a $10 \mathrm{~mm}$ epigastric trocar was used to insert the electrocautery hook, aspirator, retrieval clamp and scissors (all these tools were $10 \mathrm{~mm}$ ). Two more $5 \mathrm{~mm}$ trocars were inserted in the right subcostal region for the introduction of the retrieval clamps.

The placement of the trocars was standardized for all the patients (Figure 2A). After the trocars were inserted, the abdominal cavity is evaluated before initiating the surgical procedure. Cases perceived to be of high complexity are at this point converted to open surgery. In the rest, after dissection of the cystic infundibulum, the cystic artery is identified and sectioned between endoclips, after which the cystic duct is isolated, ligated between endoclips, and sectioned.

The dissection of the gallbladder as well as the hemostasis of the hepatic bed is performed with electrocautery. After the gall bladder is completely freed, hemostasis is confirmed and the abdominal cavity is cleaned. After transferring the optic to the epigastric portal, the gall bladder is removed through the umbilical trocar.

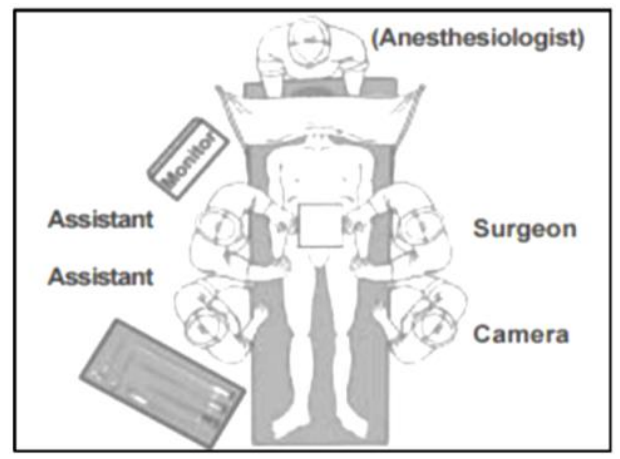

Fig.1 Positioning for the surgical equipment

\section{Mini-Iaparoscopic Cholecystectomy}

After standard positioning of the surgical team (Figure 1), the pneumoperitoneum was established by the open technique, through umbilical incision, in which a $10 \mathrm{~mm}$ trocar was inserted, using intra-abdominal pressure of 8 to $12 \mathrm{mmHg}$.

After the pneumoperitoneum was established a $30^{\circ} / 10 \mathrm{~mm}$ optic was introduced through the umbilical trocar. Given its high cost and limited durability, the $2 / 3$ $\mathrm{mm}$ mini-laparoscopic optic was not used in a single case. Three more trocars were then inserted (Figure 2B): the 3 $\mathrm{mm}$ epigastric trocar was used for the insertion of the electrocautery (hook), aspirator, retrieval clamp and scissors (all these tools were $3 \mathrm{~mm}$ ). Two more $2 \mathrm{~mm}$ trocars were inserted in the right subcostal region for the introduction of the retrieval clamps. The placement of the trocars was standardized for all patients (Figure 2B).

After the trocars were inserted, the abdominal cavity is evaluated before initiating the surgical procedure. High complexity cases at this point were converted to conventional laparoscopy with $5 \mathrm{~mm}$ trocars. In the rest, after dissection of the cystic infundibulum, the cystic artery is identified and cauterized close to it, after which 
the cystic duct is isolated, ligated and sectioned between surgical knots of 2-0 braided polyester. The dissection of the gall bladder, as well as the hemostasis of the hepatic bed is done with the electrocautery "hook". After the gall bladder is completely freed, hemostasis is confirmed and the abdominal cavity is cleaned.

A bag is improvised from the wrist of a sterile glove for the retrograde removal of the gall bladder, replacing the costly "endobag". The bag is introduced the site of the $10 \mathrm{~mm}$ umbilical trocar. The optic is reintroduced, the gall bladder is inserted in the bag and is guided by the most lateral clamp toward the optic trocar through which the removal is completed. None of the mini-laparoscopic procedures required the use of "clips", "endobags" or $2 / 3$ $\mathrm{mm}$ minilaparoscope optics.

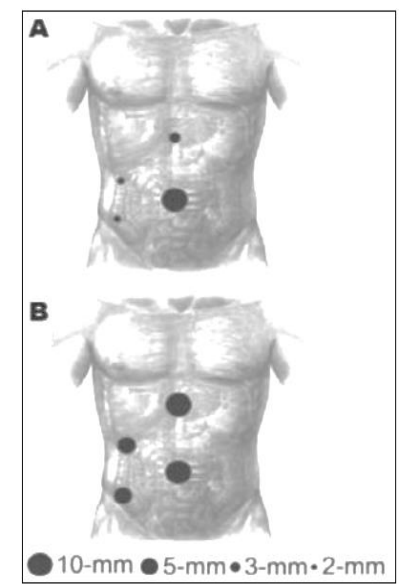

Fig.2 Trocars, A: Incisions of mini-Laparoscopy (MLS), B: Incision of the Conventional Laparoscopy (CLC)

\section{Results}

Because the operating room, medications and room charges of the hospitalization were standardized, the difference in total charges between the two groups was due to charges for surgical material, which in this case involved principally surgical trocars, clips, and sutures.

Table 2 Average hospital charges detailing the materials used in each of the two procedures

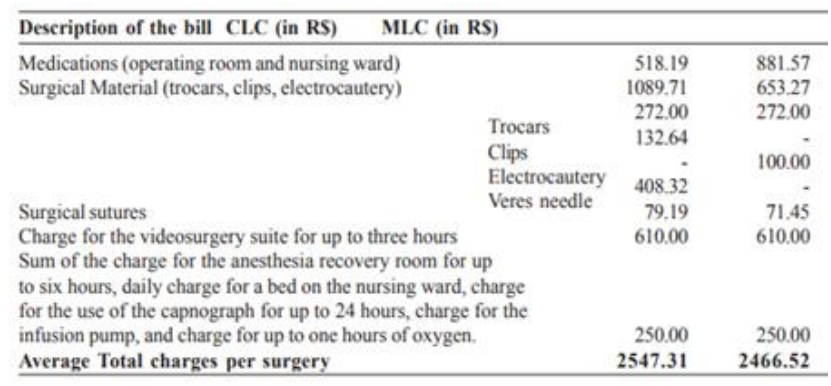

There was no statistically significant difference in the total hospital charges between the two procedures studied. For the MLC procedures there was a reduction of close to $3 \%$ of charges, when compared with the CLC procedures. While the average charge for the minilaparoscopic cholecystectomy was Rs 2,470.00, the average charge for conventional laparoscopic cholecystectomy were Rs 2,550.00.

\section{Discussion}

The standardization of several parameters was considered necessary because of factors peculiar to each patient which could interfere in the total charges of each procedure. The procedures were in a private hospital offering a variety of accommodations ranging from multibed nursing wards to private rooms with a private duty attendant. So that hospital room charges which would not be affected by patient choices in their accommodation, a standard daily room charge was applied for all cases based on the charge for a nursing ward bed without an attendant.

Other items that vary depending on individual factors and that would affect the charges were grouped and were similarly standardized for all the surgeries. This was the approach used for continuous infusion pumps, capnographs and oxygen. All cases were considered to have used one infusion pump, a capnograph for up to 24 hours, and oxygen for up to one hour during the surgery, since none of the 40 procedures lasted longer than one hour.

Others services used rarely, such as the anesthesia recovery room for more than six hours, and need for oxygen exceeding one hour, or other utilization such as emergency consultations, and laboratory tests not directly related to the surgical procedure were excluded from the calculation of individual patient's hospital charges. Regarding the surgical techniques, besides the discrepancy in the diameter of the clamps, the cases differed in relation to the utilization of endoclips.

While the conventional laparoscopic procedures studied used endoclips, the MLC used surgical sutures instead. Regarding the equipment used, those of a narrower diameter are more expensive and more delicate, but not more fragile, as the useful life of the equipment for the two groups was equal. Still, in the MLC the electrocautery hook had to be substituted every four procedures, resulting in an additional charge per surgery of approximately Rs 100.00 .

It is worth noting that the non-use of endoclips in the conventional laparoscopic procedure is a variant of this technique and can reduce the costs of the procedure. Still, in the surgeries using the minilaparoscopic technique, the average of total medication charges including anesthesia (sedation) and postoperative drugs was about $70 \%$ greater (Rs 880.00) than for the conventional laparoscopic technique (Rs 520.00).

This difference can be explained by the use of different drugs for the induction of anesthesia and different post-operative standing medication orders that were not standardized among the surgeons, factors that 
reflect the experience of the surgeon with certain drugs and peculiarities of the patients undergoing the surgeries in the series. If the charges associated with the procedure might constitute a barrier to the indication of the minilaparoscopic cholecystectomy, this study finds equivalence in the hospital charges of the two techniques.

Certainly, the cost de acquisition of the minilaparoscopy equipment should be mentioned; those of smaller diameter utilized in the minilaparoscopic procedure are a bit more costly when compared with those utilized in the conventional laparoscopic cholecystectomy [8]. But this study limited its analysis to hospital charges for the surgical procedure, after acquisition of the equipment. More studies comparing patient satisfaction with the procedures, parameterization of pain and return to normal activities are necessary for a more detailed analysis of the indications of these procedures.

\section{Conclusions}

Because it does not represent an increase in hospital charges when compared to the conventional laparoscopic procedure, the mini-laparoscopic cholecystectomy should be more widespread and more frequently indicated by surgeons. Besides the similarity in charges, the superior cosmetic benefits of mini-laparoscopic cholecystectomy tiny orifices resulting in imperceptible scars.

\section{References}

[1]. Carvalho GL, Silva FW, Cavalcanti CH, Albuquerque PC, TG, et al. Minilaparoscopic cholecystectomy Sem. 2007; 5(1):511.
[2]. Gagner M, Garcia-Ruiz A. Technical aspects of minimally invasive abdominal surgery performed with needlescopic instruments. Surg Laparosc Endosc. 1998; 8:171-9.

[3]. Lee PC, Lai IR, Yu SC. Minilaparoscopic (needlescopic) cholecystectomy: A study of 1,011 cases. Surg Endosc. 2004; 18:1480-1484.

[4]. Look SP, Chew YC, Tan SE, Liew DM, Cheong JC, Tan SB, et al. Post-operative pain in needlescopic versus conventional laparoscopic cholecystectomy: a prospective randomised trial. J R Coll Surg Edinb. 2001; 46:138-142.

[5]. Mamazza J, Schlachta CM, Seshadri PA, Cadeddu MO, Poulin EC. Needlescopic surgery a logical evolution from conventional laparoscopic surgery. Surg Endosc. 2001; 15:1208-1212.

[6]. McCloy A, Randall D, Schug SA, Kehlet H, Simanski C, Bonnet $F$, et al. Is smaller necessarily better? A systematic review comparing the effects of minilaparoscopic and conventional laparoscopic cholecystectomy on patient outcomes. Surg Endosc. 2008; 22:2541-2553.

[7]. Ros A, Gustafsson L, Krook H, Nordgren CE, Thorell A, Wallin G, et al. Laparoscopic cholecystectomy versus minilaparotomy cholecystectomy a prospective, randomized, single-blind study. Annals of Surgery. 2001; 234(6):741-749.

[8]. Siddiqui T, MacDonald A, Chong PS, Jenkins JT. Early versus delayed laparoscopic cholecystectomy for acute cholecystitis: a meta-analysis of randomized clinical trials. The American Journal of Surgery. 2008; 195:40-47.

[9]. Squirrell DM, Majeed AW, Troy G, Peacock JE, Nicholl JP, Johnson AG. A randomised, prospective, blinded comparison of post-operative pain, metabolic response, and perceived health after laparoscopic and small incision cholecystectomy. Surgery. 1998; 123:485-95.

[10]. Visser BC, Parks RW, Garden OJ. Open cholecystectomy in the laparoendoscopic era. The American Journal of Surgery. 2008; 195:108-114. 